\title{
Applications of Fiber Optic Sensors in Traffic Monitoring: A Review
}

This paper was downloaded from TechRxiv (https://www.techrxiv.org).

LICENSE

CC BY 4.0

SUBMISSION DATE / POSTED DATE

$15-02-2022$ / 22-02-2022

CITATION

Tekinay, Mustafa; Ganesh, Thiagarajan; Brunton, Matthew; Sylvester, Tim (2022): Applications of Fiber Optic Sensors in Traffic Monitoring: A Review. TechRxiv. Preprint. https://doi.org/10.36227/techrxiv.19178267.v1

$\mathrm{DOI}$

10.36227/techrxiv.19178267.v1 


\title{
Applications of Fiber Optic Sensors in Traffic Monitoring: A Review
}

\author{
Mustafa Tekinay, Tim Sylvester, Matthew Brunton, and Thiagarajan Ganesh
}

\begin{abstract}
Fiber optic sensors are becoming key tools in building more robust and accurate traffic monitoring systems. In this survey, we present various preliminary implementations and a discussion about some of the key design points. The scanning rates of the sensors should be at least $500 \mathrm{~Hz}$ to correctly capture the moving vehicles. The size of the sensors should not be larger than approximately $50 \mathrm{~cm}$ to enable individual axle detection. A minimum of $1 \mu \epsilon$ sensor resolution is required to detect light weight vehicles. Adaptive filtering, Wavelet denoising and neural networks are some of the tools used to improve the performance. Sensors that reside on the surface of the pavement might lead to biased data and cause destructive implementation. There is yet to be a unified solution. We need a global, user-friendly, consistent, durable, environment-friendly solution with high system availability that would work on bridges, in urban areas and in tunnels. We believe precast slabs of road pavement embedded with sensors that are manufactured at a facility would give us the best opportunity to meet those demands.
\end{abstract}

Index Terms_-fiber Bragg grating sensors, fiber optic sensors, smart infrastructure, smart roads, strain based sensing

\section{INTRODUCTION}

$\mathbf{I}^{2}$ NTELLIGENT road pavement and real-time traffic management are emerging as parts of the smart infrastructure industry. Monitoring and managing the real-time traffic flow can help in adjusting traffic signal control and traffic regulation, reducing traffic congestion, and detecting atypical behavioral events [1]. Perhaps, it can also supplement the next generation of self-driving vehicles so that we can have a true connection between smart vehicles and smart pavement. Technologies utilizing video cameras, radars, magnetic fields, and wireless sensors are currently used to monitor traffic flow. However, these methods are expensive and difficult to install and maintain. They are also easily damaged and influenced by the environment.

In order to overcome the aforementioned difficulties of traffic monitoring; unified, cost-efficient, accurate, consistent, and safe solutions are needed. Fiber optic sensors (FOSs) have been implemented in various ways to serve this purpose. In this survey, we highlight these implementations in practice with their advantages and limitations, identify essential design parameter values for a successful system, and suggest a scalable solution.

Mustafa Tekinay and Tim Sylvester are with Integrated Roadways LCC, 1712 Main St. Ste. 2007, Kansas City, MO 64108, USA (e-mail: \{mustafa, tim\}@integratedroadways.com).

Matthew Brunton is with Monitor Optics Systems Ltd., 8 / 303 Pacific Highway, Lindfield, NSW 2070, Australia (e-mail: mbrunton@monitoroptics.com).

Thiagarajan Ganesh is with the Department of Civil and Mechanical Engineering, University of Missouri-Kansas City, Kansas City, MO 64110, USA (e-mail: ganesht@umkc.edu).
The remainder of this paper is organized as follows: In Section II, fundamentals of FOSs are presented. Section III reviews various real-world implementations of FOSs. Analysis of the outcomes is given in Section IV. Section V concludes the survey.

\section{FIBER OPTIC SENSORS}

Over the last few decades FOSs have gained increasing attention from researchers and engineers in various fields. They are accurate, durable, immune to electromagnetic interference, require relatively less power, and have relatively low lifetime cost [2]-[4]. Due to these virtues, they are outperforming the conventional electrical sensors such as piezoelectric sensors and strain gauges. Understanding the physical characteristics of light waves and their interactions with surroundings have led to designs that act as strain, temperature, acoustic, magnetic fields, acceleration, rotation, pressure, humidity and viscosity sensors. They are employed in a wide range of industries such as oil and gas, energy, biomedical, civil, aerospace, and transportation [5], [6].

Basic principle of all FOSs is to detect variations in intensity, phase, frequency and polarization of light waves induced by the measured variable. Two key performance metrics of FOSs are sensitivity and resolution. Sensitivity represents the relationship between the variation of the sensor output and the corresponding variation in measured variable. A good sensor should have a high sensitivity, i.e., small variations in the measured variable should correspond to large variations in the output. Resolution describes the sensor's ability to detect 
small variations. It is defined as "the variation in the value of the measured variable that causes a variation in the output value equal to the uncertainty of the output itself" [5]. In other words, any variations less than the resolution value can not be accurately sensed.

FOSs can be categorized into three types: single-point, quasi-distributed (multiplexed), and distributed sensors (see Fig. 1). Quasi-distributed sensors are essentially point sensors that are multiplexed and located on pre-determined locations.

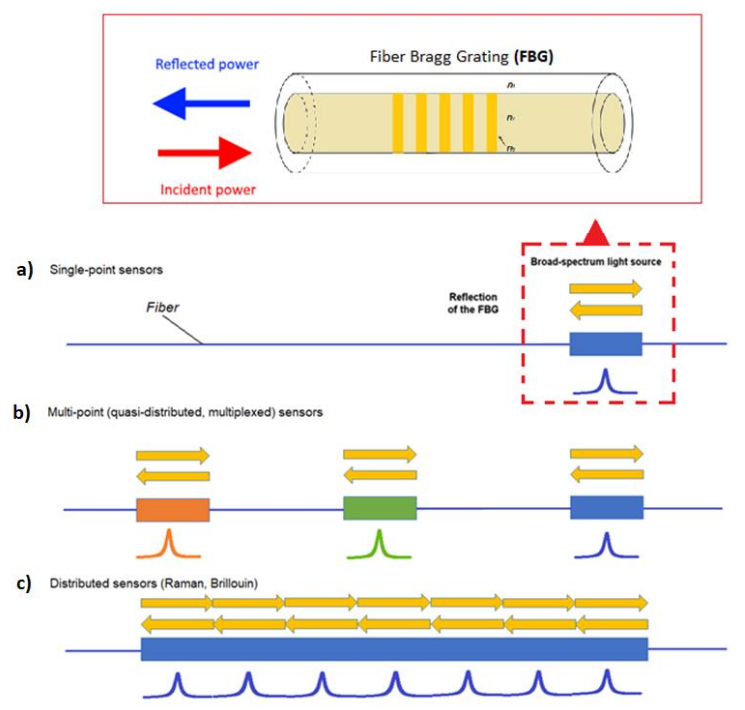

Fig. 1: Fiber optic sensor categories: (a) single-point, (b) multiplexed, and (c) distributed [5].

\section{A. Single-point sensors}

The most straightforward single-point sensors are based on interferometry. Interferometer sensors, as the name suggests, exploit the interference between light beams. In an interferometer, the light is separated into two beams where one beam is subjected to the sensing environment experiencing a phase shift, while the other is used as a reference preserved from the sensing environment [7]. The beams then are recombined. This results in either constructive or destructive interference. Monitoring the recombined beam yields information about the measured variable. Fabry-Perot, Michelson, Mach-Zender and Sagnac interferometers are some of the well known configurations. A comprehensive review of interferometric sensors is presented in [8], [9].

There are also grating based single-point sensors such as fiber Bragg grating (FBG) and long period grating. A typical FBG has a sub-micron period whereas a long period grating has a period in the range $100 \mu \mathrm{m}$ to $1 \mathrm{~mm}$ [10]. A thorough review of long period grating sensors is given in [10], [11].

FBG based sensors occupy a large market share due to a number of advantages: small form factor, lightweight, selfreferencing, easy to multiplex, no need for electrical connections and the compatibility for non-invasive remote sensing [5]. Self-referencing is a very important strength because the output does not directly depend on the total light intensity or losses in the fibers and couplers. The output of an FBG sensor is given in terms of an absolute parameter (wavelength) [8]. This property makes FBG sensors more robust, accurate, and relatively simple to operate.

The basic working principle of an FBG is illustrated in Fig. 2.

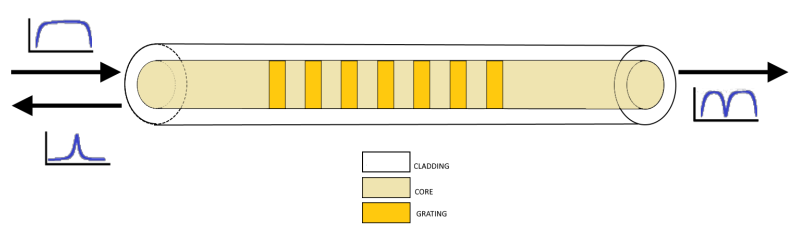

Fig. 2: The grating reflects some of the transmitted light beam back. The plots represent the spectrum of the incident, reflected, and transmitted beams [5].

A broad-spectrum light beam is transmitted through a fiber cable with an FBG. The FBG reflects a subset of the broadspectrum light back to the source and transmits all other light. The resulting back-propagating spectrum presents a peak centered at the Bragg wavelength. The spectrum of the beam that passed through resembles the spectrum of a notch filter. The Bragg wavelength is dependent on the grating pitch, which is a design parameter, and the refractive index of the fiber. The reflected Bragg wavelength, $\lambda_{B}$, is defined as:

$$
\lambda_{B}=2 n_{e f f} \Lambda
$$

where $n_{\text {eff }}$ and $\Lambda$ denote the effective refractive index and the grating pitch (periodic spacing of the grating) respectively. A variation in the environmental parameters, for instance, temperature and strain, influence both the pitch $(\Lambda)$, and the refractive index $\left(n_{\text {eff }}\right)$. This causes a shift in the reflected spectrum (see Fig. 3). In sensing applications, the Bragg wavelength (central frequency of the reflected spectrum) is monitored.

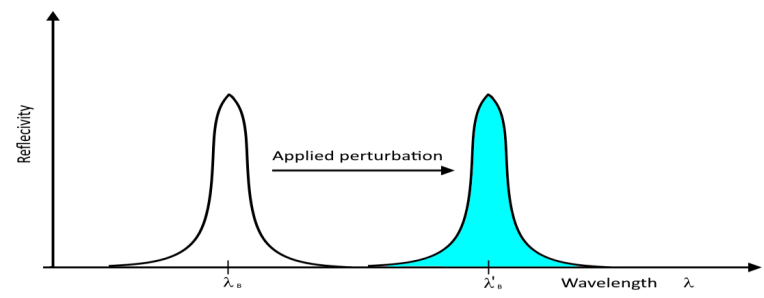

Fig. 3: Shift in the Bragg wavelength induced by a strain or temperature [5].

Typically, a Bragg wavelength written into a single mode fiber SMF28 will shift approximately $1.2 \mathrm{pm} / \mu \varepsilon$ [5]. Temperature change also causes variation in the effective refractive index and the grating pitch due to thermal expansion. A typical temperature induced Bragg wavelength shift is approximately $13.7 \mathrm{pm} /{ }^{\circ} \mathrm{C}$. Therefore, it is important to consider the temperature dependence of the sensor output and devise methods to compensate for it.

The accuracy of measurement and the number of measurements per second are dependent on the optical interrogation 
unit. The interrogator provides the broadband light source and also tracks the reflected peak wavelengths in real time. Interrogator wavelength resolution can be as low as $1 \mathrm{pm}$, which equates to a $0.83 \mu \varepsilon$ measurement resolution, at acquisition frequencies up to $1000 \mathrm{~Hz}$ [12]. The resolution reduces to around $3 \mathrm{pm}$ at $5000 \mathrm{~Hz}$ acquisition frequency.

\section{B. Distributed sensors}

Distributed sensors have the ability to sense at any point along the fiber cable through scattering. When a light beam is transmitted into a fiber optic cable it experiences random Rayleigh, Brillouin and Raman scattering along the way and some of the scattered signal travel backwards. These scatterings arise from the interaction of the light waves with the particles within the fiber and with fiber glass. Fluctuations in the density of the fiber glass and thermally generated acoustic waves change the properties of these backscattered waves. The local changes in temperature, strain, and vibration along the cable will modulate the signal. Monitoring the changes in the backscattered signals yields information about the measured variable. The backscattered signals are mapped to locations along the cable depending on their arrival time to the origin. The smallest length within which a significant change in the measured variable can be detected is defined as the spatial resolution [5]. The concept of a distributed sensor is shown in Fig. 4. Acquisition frequency and accuracy vary between the different technologies, and will also depend on the length of the fiber, desired spatial resolution, and the time taken to make the measurement.

Physics of distributed fiber optic sensors are presented in great detail in [13], [14].

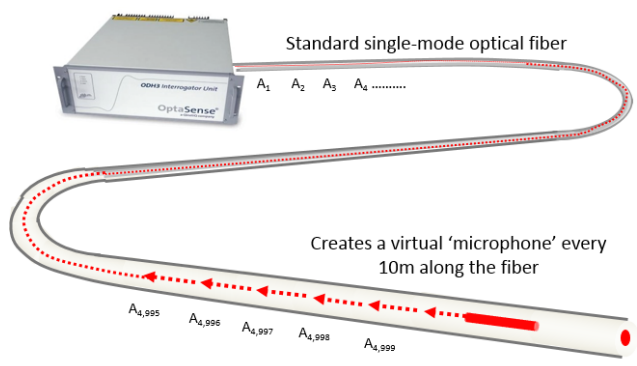

Fig. 4: Distributed FOSs [15].

\section{IMPLEMENTATIONS}

FOSs have been utilized in various experiments dating back to late twentieth century to demonstrate their excellent potential for traffic monitoring. Mimbela et al. [16] provide a comprehensive review of some of these earlier projects that laid the foundation for the next generation implementations. Here, we present some of the recent developments in the field.

\section{A. Bridges}

1) Concrete bridge in Northern Ireland: Lydon et al. [17] equipped a reinforced concrete bridge in Loughbrickland, Northern Ireland with FBG sensors (10 mm length) to monitor deterioration of the structure, weigh individual vehicles and detect overweight vehicles (see Fig. 5).

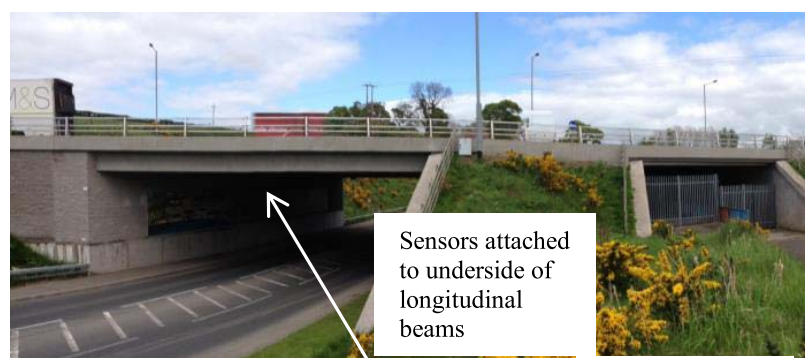

Fig. 5: The concrete bridge in Loughbrickland, Northern Ireland [17].

The authors suggest that placing the sensors underneath the bridge is a better solution then placing them on the road pavement in terms of longevity and portability of the system. Their finite element analysis (FEA) showed that an overloaded 6 axle truck produced $30 \mu \epsilon$, a light goods vehicle produced about $5 \mu \epsilon$ and a compact car on the slab soffit/deck produced about $1 \mu \epsilon$. After running trials, they found out that slab soffit strain values decrease if the vehicles travel directly over the girder. To address this problem, they placed FBGs on the girders in addition to the soffit. The consequent improvement to the axle detection accuracy is presented in Fig. 6.
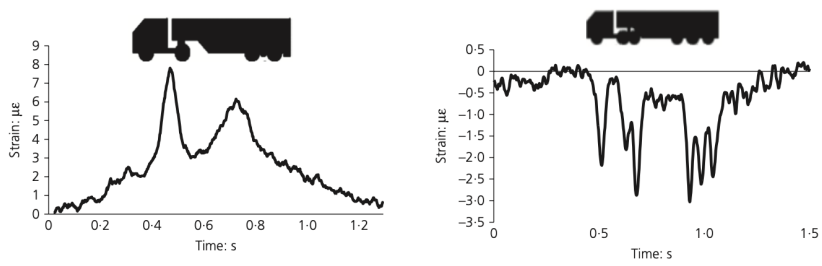

Fig. 6: Axle detection accuracy improvement; soffit sensor (left) vs girder sensor (right) [18].

One can see that the signal from the girder compared to the soffit has more distinguishable peaks.

Another challenge they faced was to distinguish individual vehicles when multiple vehicles travel side by side on different lanes [19]. They included more sensors in the transverse direction and developed post processing algorithms to overcome this. The system also included a separate FBG to compensate for temperature strain.

After calibrating the system, they compared their weight estimations with the values obtained from a nearby static weighing station. Average individual axle weighing error was $-2.25 \%$ and average gross vehicle weighing error was $-4.5 \%$. The authors think that the regular under-weighing might be due to a signal processing error [20]. Calibration with different vehicle types (as opposed to one) at different speeds is anticipated to enhance the accuracy as well.

During their investigation, the authors also learnt that FBG sensors perform better than conventional strip sensors, electrical resistance sensors, and vibrating wire sensors due to various reasons. Temperature effect seems to be the most concerning problem for conventional strip sensors and electrical 
resistance sensors. Moreover, in order to install conventional strip sensors one needs to destruct the road pavement. Vibrating wire sensor produces accurate results at a $0.2 \mathrm{~Hz}$ scanning rate. This is a very low scanning rate for live traffic data acquisition. The minimum scanning rate should be around 500 - $1000 \mathrm{~Hz}$.

2) Steel girder bridge in LaGrange, Georgia: In a recent work, Oskoui [21] presented a weigh-in-motion (WIM) system on a four-span steel girder bridge in LaGrange, Georgia (see Fig. 7).

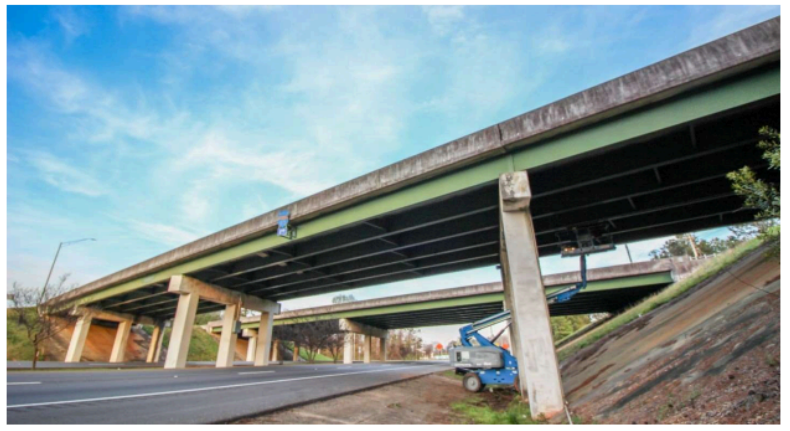

Fig. 7: The steel girder bridge in LaGrange, GA [21].

The goal of the system is to weigh vehicles, estimate axle spacing and speed. The proposed method requires two wheel sensors for axle spacing and speed calculations and a rotation sensor for axle weight calculations. They employ FBG sensors with $75 \mathrm{~mm}$ gauge lengths. The wheel sensors were placed directly under the deck at at a transverse position where a wheel is likely to go directly over. The FBG sensor that is meant to measure the rotation at the bridge abutment was installed on a cantilever beam as shown in Fig 8 .
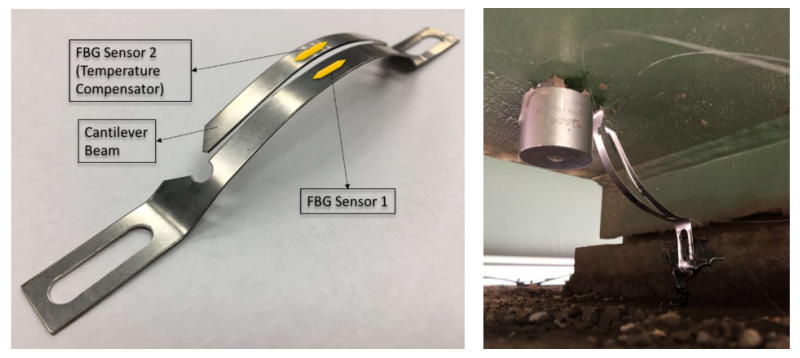

Fig. 8: Rotation sensor [21].

Temperature strain is compensated with a dedicated sensor that is placed on the arm of the cantilever beam with an open end. Bridge installation positions of the sensors are shown in Fig. 9.

The abutment rotation, hence strain, values are calibrated using 4 trucks with known axle weight and spacing. The estimated vehicle weights are compared with the values obtained from a nearby static weighing station. Out of 10 field trials, absolute individual axle weighing error values ranged from $0.23 \%$ to $16.67 \%$ with a mean value of $-3.29 \%$. Absolute gross vehicle weight $(\mathrm{GVW})$ estimation error ranged from $0.06 \%$ to $12.08 \%$ with a mean value of $-3.38 \%$. Axle spacing estimations were within $3 \%$ error.
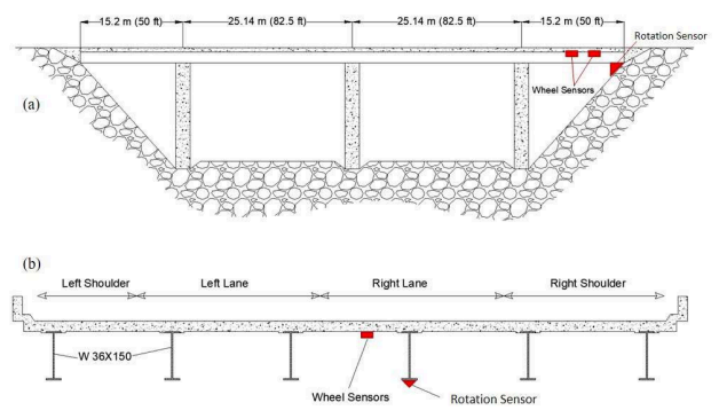

Fig. 9: Sensor locations [21].

3) Longweigang Bridge in Jingdezhen, China: Long-gauge FBG sensors are utilized on the Longweigang Bridge as a part of a WIM system. The Longweigang Bridge is a simply supported reinforced concrete plate girder bridge. Chen et al. [22] argue that long gauge FBGs are more immune to cracks and damages on a structure compared to point sensors. They installed four long-gauge $(0.5 \mathrm{~m}$ gauge length) $\mathrm{FBG}$ sensors on the plate girder of a bridge in Jingdezhen, China as shown in Fig. 10. The distance between each sensor was $2 \mathrm{~m}$.
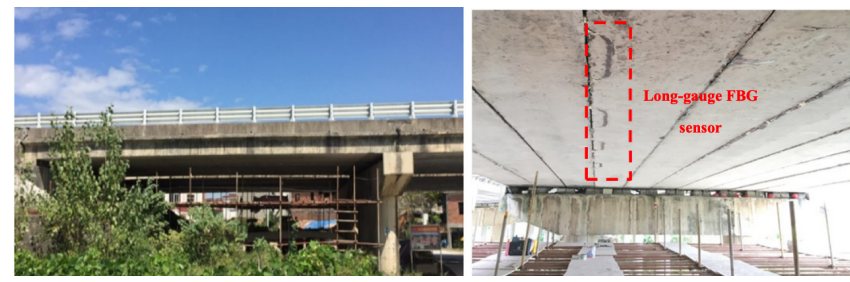

Fig. 10: FBG installation on the Longweigang Bridge in Jingdezhen, China [22].

The calibration was carried out by a heavy truck with a gross weight of $24,929 \mathrm{~kg}$ and three axles. The validation trials were done with 5 vehicles; one SUV, one bus and three trucks. The error values were about $4 \%, 4 \%$ and $7 \%$ for velocity, wheelbase and GVW respectively. The errors were a bit higher than their theoretical results. They think this might be because of the higher noise level in an actual environment. The main limitation of this design was that any wheelbase smaller than the $2.5 \mathrm{~m}$ could not be identified due to the larger gauge length.

4) Concrete bridge in Sacramento, California: This project included a prestressed box-girder bridge in Sacramento, California and a set of FBG rosettes. Bao et al. [23] focused on capturing the shear strain near the abutment and comparing it to traditional flexural strain. The installed rosette sensor was made of two strain FBGs (75 mm gauge length) placed at an angle. Since the shear strain is subtraction of the strain value from one sensor from other, any temperature strain cancel each other out, yielding a self temperature-compensating shear strain sensor. Both of the sensors were installed inside the concrete box-girder. The bridge and the placements of the two rosettes are shown in Fig. 11.

The calibration tests were carried away at night time with a dump truck with a trailer. The superiority of the shear response to the flexural response in terms of distinguishing individual 

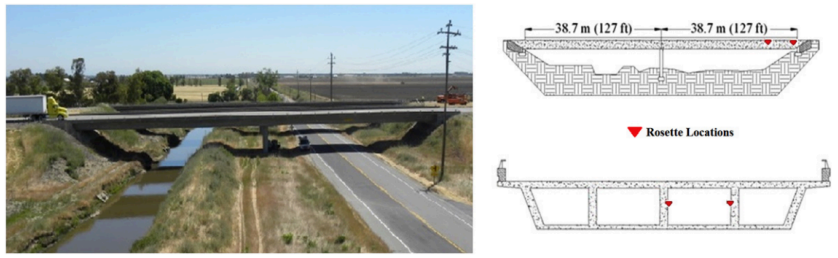

Fig. 11: Concrete bridge in Sacramento, California and sensor placements [23].

axles is demonstrated in Fig. 12.

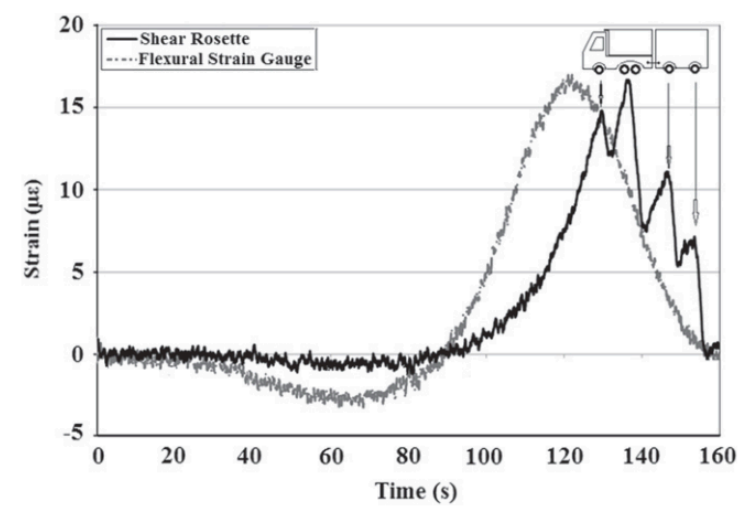

Fig. 12: Shear and flexural response of the bridge [23].

This is because the flexural strain is the result of overall vehicle weight whereas shear strain is more sensitive to individual axle weights. Even though shear rosettes can distinguish more axles, they also fail when the wheelbase is very small (tandem axle) as seen in the figure. Absolute axle weighing errors ranged from $0.4 \%$ to $18.5 \%$. Maximum GVW and axle spacing errors were $1.5 \%$ and $11.9 \%$ respectively out of 3 samples. Similar tests were also carried out in Elmhurst Illinois and Chicago, Illinois [23].

5) Hampden Bridge in New South Wales, Australia: The Hampden Bridge is a heritage listed bridge located in New South Wales, Australia. It is a steel suspension bridge that runs across the Kangaroo River (see Fig. 13). In 2007, the local traffic authority suspected that vehicles heavier than the bridge's load limit were using the bridge following an observed increase in unscheduled maintenance requirements. To understand whether overweight vehicles were using the bridge, Monitor Optics Systems installed FBG sensors onto key suspension rod members [24].
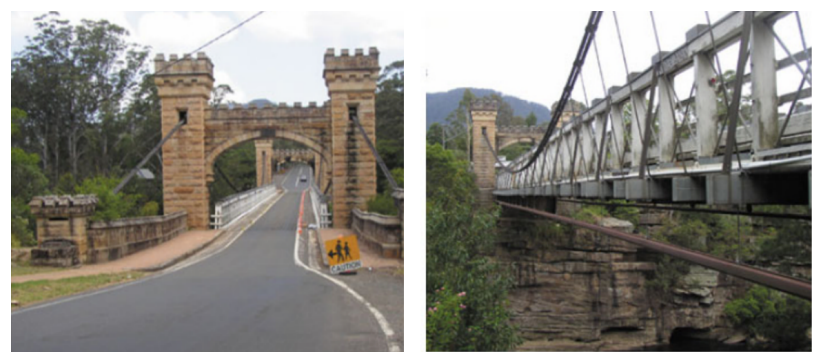

Fig. 13: Hampden Bridge in New South Wales, Australia [24].
The FBG sensors were attached to two key suspension rod members that connected to the bridge's hinge joint in the center. This allowed a linear strain to load relationship, and thus creating a WIM bridge. A truck that weighed $42,500 \mathrm{~kg}$ (the bridge's load limit) was used to calibrate the sensors, driving over the bridge from each direction 3 times (total of 6 tests). The strain recorded for each test was approximately $350 \mu \varepsilon$, with a total of $5 \%$ variation in the strain over the 6 tests. The recorded strain for a test is given in Fig. 14.

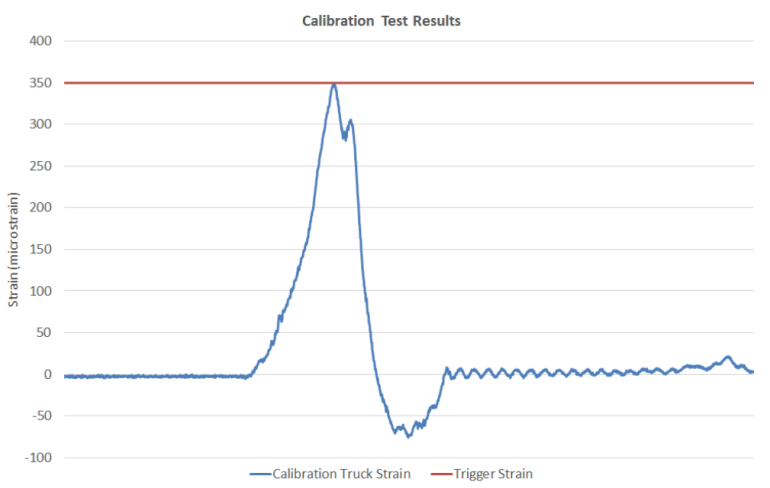

Fig. 14: Recorded strain response of a 42,500 kg truck [24].

Following a successful trial period, cameras were then implemented onto the bridge to capture the details of the offending vehicles. The strain data and vehicle pictures were sent to an online database that the local traffic authority could monitor.

6) Bridge prototype: Alamandala et al. [3] built a $250 \mathrm{~cm}$ long and $30 \mathrm{~cm}$ wide bridge prototype and equipped it with two FBG sensors. The prototype has five beams and six pairs of $12 \mathrm{~cm}$ high piers. A robotic vehicle is used in their tests. Their report indicates that there is a linear relationship with roughly 0.9 linear coefficient between the weight of the vehicle and shift of the Bragg wavelength. The maximum individual axle weight estimation error is roughly $25 \%$ out of 15 samples.

\section{B. Road pavement}

1) Road surface in Riga, Latvia: Grakovski et al. [25], [26] placed fiber optic cables by Sensor Line in $30 \mathrm{~mm}$ wide gaps on the surface of the road in Riga, Latvia. The gaps were filled with resilient rubber afterwards to complete the installation. This is illustrated in Fig. 15.
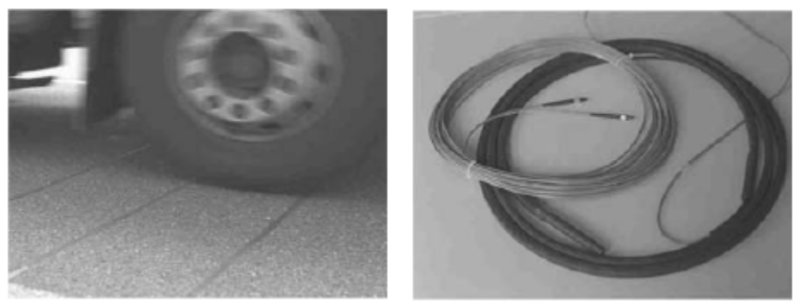

Fig. 15: Pavement cable installation in Riga, Latvia [25].

They used a loaded truck with speed ranging from 10 to $90 \mathrm{~km} / \mathrm{h}$. The field trials showed that the wheel size, wheel pressure and speed changed the force applied to the ground due 
to inertia properties. They estimated the wheel footprint (area of the wheel contact), speed, and axle weights. For typical wheelbase values, the method achieved accuracy within $4 \%$ for footprint. For tandem axles, the method needs to be improved as the error was $31 \%$ for one of the footprint estimations. For speeds above $48 \mathrm{~km} / \mathrm{h}$, the axle weighing errors were typically within $10 \%$. However at lower speeds, errors were in the range of $10 \%-20 \%$. The authors believe that the effects of acceleration and deceleration became more apparent in the signals at lower speeds. Specifically, the vertical vibration of the vehicle was attributed as the reason for this. To mitigate this, they suggest using a platform to increase the exposure time of the load to the sensors.

2) Concrete road in Otsego, Minnesota: Al-Tarawneh [27] presented a proof of concept for traffic monitoring using FBGs at a research facility in Otsego, Minnesota. The experiments were carried out at section Cell 40 of the test road. The site is shown in Fig. 16.

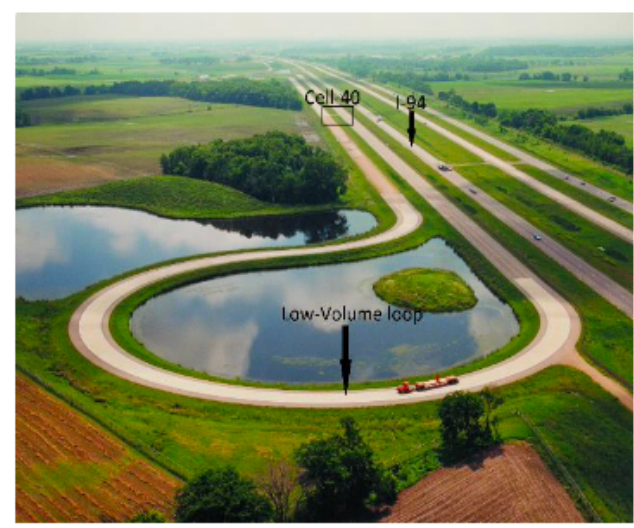

Fig. 16: Cold Weather Research Facility in Otsego, Minnesota [27].

He embedded a 3-D FBG (see Fig. 17) inside a concrete panel approximately $2.54 \mathrm{~cm}$ (1 inch) deep along a likely wheel-path. Glass fiber reinforced polymer material was used for protection. The 3-D sensor captures vertical, longitudinal and transverse strains.

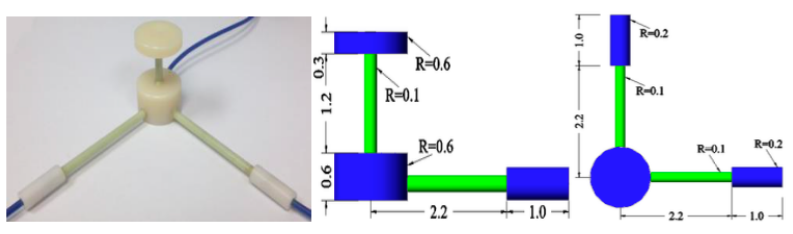

Fig. 17: The 3-D FBG sensor [27].

Calibration tests were carried out by a 5 -axle semi-truck with GVW of $36,290 \mathrm{~kg}$. Results yielded that the longitudinal component of the sensor, compared to vertical and transverse ones, showed the most sensitivity and produced the most reliable results. Longitudinal sensor achieved an average weight measurement accuracy of $90 \%$. Transverse and vertical sensors achieved $79 \%$ and $67 \%$ respectively. The performance discrepancy is believed to be due to the transverse position of the wheel. Vertical and transverse sensors required the wheel to be (almost) directly over the sensor whereas the longitudinal sensor was not as sensitive to that.

Al-Tarawneh also theoretically showed that in a nonreinforced slab of concrete, the sensitivity was maximum toward the top (surface of the slab) and bottom in terms of depth. The sensitivity was minimum in the middle portion of the slab. Moreover, it was found that smaller slab surface area leads to better sensitivity for the sensors. This is especially true when the slab size is smaller than $1.83 \mathrm{~m}$ (6 feet). Increasing the slab length beyond $1.83 \mathrm{~m}$ (6 feet) has an insignificant effect on the sensor sensitivity. To achieve $80 \%$ WIM accuracy with the longitudinal sensor, the center of the wheel should be within $15.24 \mathrm{~cm}$ (6 inches) around the sensor. Based on an analytical model for modulus elasticity of the concrete pavement, it was shown that after 10 years, the sensitivity of the sensor will drop by $10 \%$ which might introduce measurement errors.

3) Asphalt road in Rotterdam, Netherlands: In Rotterdam, Karabacak et al. [28] laid down two FBG equipped fiber optic cables that run perpendicular to the direction of travel and are $1.5 \mathrm{~m}$ apart. The cables are $15.24 \mathrm{~cm}$ (6 inches) deep and each host an array of FBGs that are $7.62 \mathrm{~cm}$ (3 inches) apart. This is illustrated in Fig. 18. They repeated the experiments at three different locations. Typical installation takes about 2-3 hours of road closure.
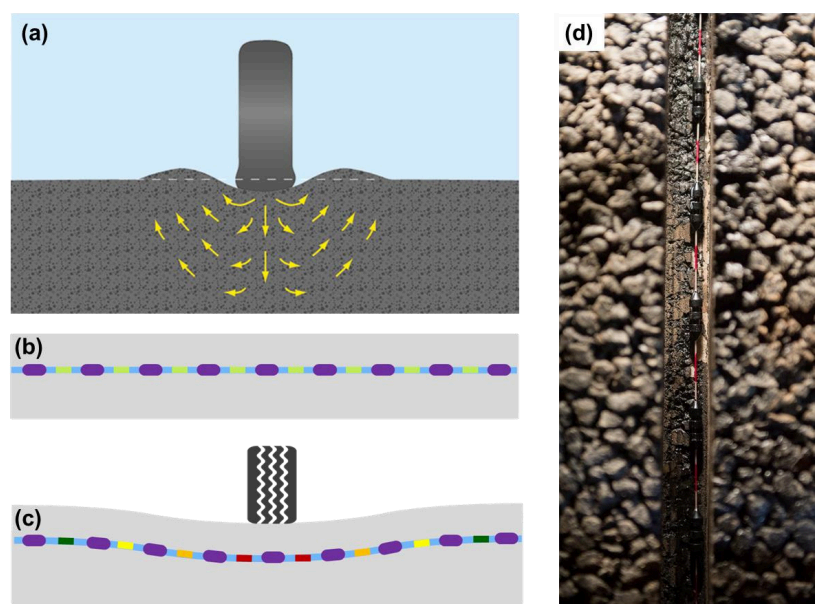

Fig. 18: Fiber installation in $15 \mathrm{~mm}$ slits in Rotterdam, Netherlands [28].

They performed tests with trucks and compact cars with speeds up to $90 \mathrm{~km} / \mathrm{h}$. A compact car with approximately $272.16 \mathrm{~kg}(660 \mathrm{lbs})$ per wheel load, generated $4 \mu \epsilon$. Local pavement structural variables, asphalt temperature, speed, and transverse wheel position are found to be key variables that affect the performance of the system. To mitigate undesirable effects by structural variables, temperature, and speed, a detailed calibration is needed. Temperature also needs to be tracked independently. The problem they faced regarding the wheel position was because of the gaps between the sensors. If the wheel was in between sensors rather than directly on top, the weight estimations were different. Therefore, calibrations could not be done precisely and that caused inconsistent 
estimations. Signal processing algorithms were devised to address this. To assess the performance of their algorithm, they compare the estimated position to a theoretical model. This comparison is illustrated in Fig. 19.
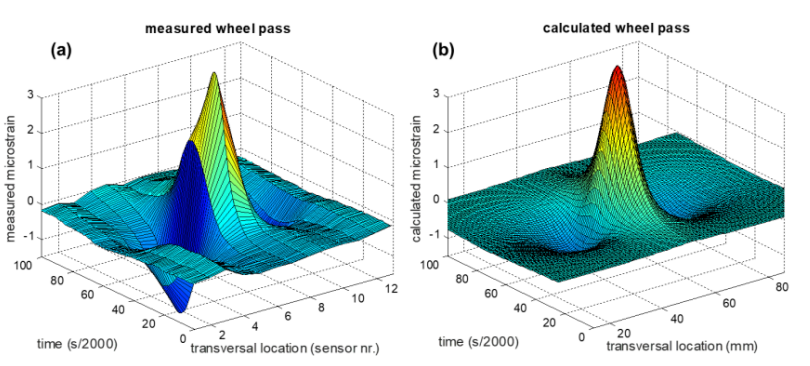

Fig. 19: Measured vs theoretical wheel position [28].

This also allows the design to calculate the track width of the vehicles. Overall performance of the WIM was C-class according to the COST323 model. The sensitivity was high enough that even a bicycle wheel was identified.

4) Asphalt road in Ostrava, Czech Republic: This project employs FBG sensors fixed into the road as well. Fajkus et al. [29] chose to house the sensors in polydimethylsiloxane (PDMS), a polymeric two-component potting material due to its flexibility and strength. A 3-D printer was used for the potting process. A potted sensor and its asphalt implementation are given in Fig. 20.
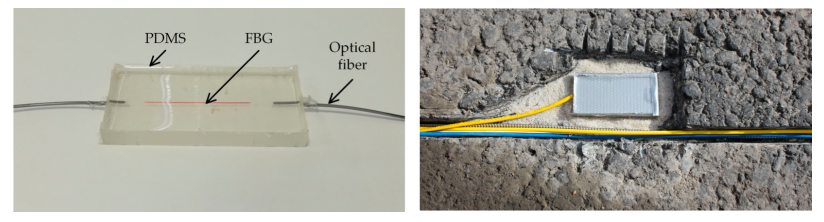

Fig. 20: Potted FBG and in-pavement installation [29].

The sensor was placed at a depth of $15 \mathrm{~mm}$. The groove was then filled with cold asphalt. During the curing process, it was found that the Bragg wavelength increased but did not impair the functionality of the sensor. Based on the sensitivity analysis, it was established that 5 embedded sensors in a chain would cover the entire lane with a width of $3.2 \mathrm{~m}$.

A couple of fiber optic cables (with 5 FBGs each) were installed in a road in Ostrava, Czech Republic $2 \mathrm{~m}$ apart. Live traffic measurements were taken for 18 days. The system's vehicle count was compared to an on-site camera detection system. The embedded sensors identified 3,963 vehicles missing only 16 vehicles (99.62\%). This a notable accuracy level. We believe that the design's high sensitivity (a car wheel inducing a $20 \mathrm{pm}$ Bragg wavelength shift) is the main factor for this. The estimated vehicle speeds were compared to the reference speeds obtained by optical gates. Axle speeds were averaged to estimate the vehicle speed. Out of 3,963 vehicles, the measured speed values ranged from $38 \mathrm{~km} / \mathrm{h}$ to $64 \mathrm{~km} / \mathrm{h}$. The maximum absolute speed estimation error was $5.83 \%$. The average error was $2.65 \%$.

5) Portable Platform in Brussels, Belgium: Yuksel et al. [30] designed a portable platform instrumented with FBGs (3 $\mathrm{mm}$ long) to monitor traffic. Typical installation is shown in Fig. 21.
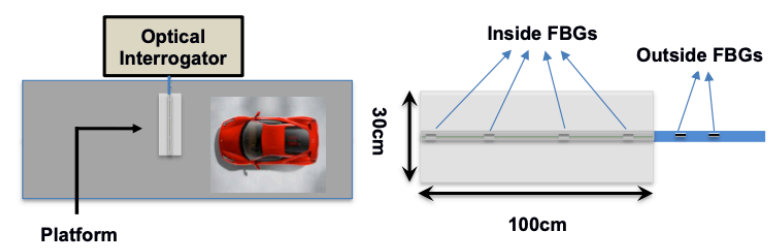

Fig. 21: Installation layout in Brussels, Belgium [30].

There are a total of 6 FBGs where 4 of them are hosted in between the metallic plates and the other 2 are out of the platform. The extra two FBGs are used for temperature compensation. All of the sensors reside in a protective tube. The height of the platform is approximately $3 \mathrm{~cm}$.

They tested their proof of concept on a road with live traffic in Brussels. In their preliminary experiments, more than 50 cars were detected in 4 hours. It is inherently challenging for the design to estimate speeds as the wheelbase information needs to be known a priori. This is still work in progress and further testing and enhancements are needed to quantitative results.

6) Portable Dynamometer: Belitsky et al. [31] propose to use a fiber optics dynamometer based on photoelasticity to identify wheel presence. The dynamometer they use is essentially made of two plates glued to each other and a singlemode optical fiber that is looped in between those plates. The plates are made of two layers; a metallic external and a rubber internal layer. They used a plastic covering for the cable. The overall thickness of the plates is approximately 1 $\mathrm{cm}$. An example installation where the plates are fastened to the ground is shown in Fig. 22.

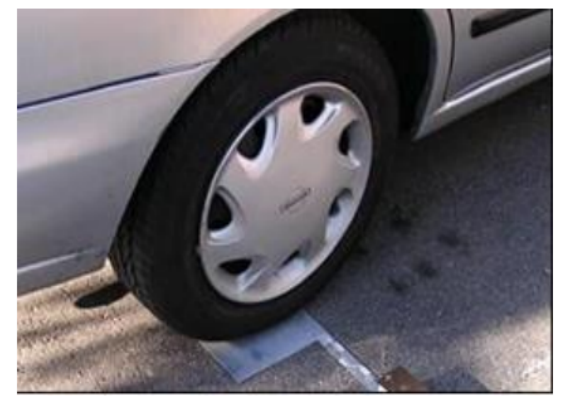

Fig. 22: Dynamometer based on photoelasticity [31].

The external vertical force on the plate modulates the light and the number of periods of the output signal changes according to the absolute value of the force. The scale's resolution is roughly about $0.33 \%$ of the vehicle's weight. To reduce the errors caused by the wheel impact on the plates (as the plates are $1 \mathrm{~cm}$ above the pavement surface), they recommend installing the plates on speed bumps. They calculate that for a vehicle with speed $50 \mathrm{~km} / \mathrm{h}$, the measurement deviation is less than $3 \%$. A temperature compensation is suggested as it affects the photoelasticity of the fiber optic material. 
Lab experimentation showed promising results (as low as $3 \%$ error).

\section{Roadside}

1) Test road in the UK: Hall and Minto [15] propose to utilize the existing spare roadside fiber optic cables as distributed acoustic sensors with minimal overhead. Their preliminary tests indicate that cars can be detected up to $30 \mathrm{~m}$ offset from the road. Although the best results are obtained when the offset distances are shorter than $10 \mathrm{~m}$. The recommended burial depth for cables is between $10 \mathrm{~cm}$ and $50 \mathrm{~cm}$. The spatial resolution of their distributed sensor is $10 \mathrm{~m}$. They suggest two separate roadside cables on each side for divided roadways (see Fig. 23).
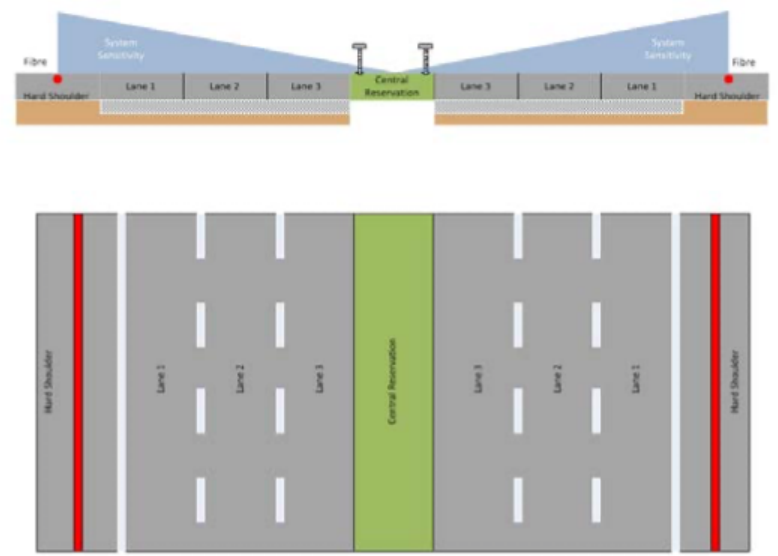

Fig. 23: Test road roadside fiber cable deployment in the UK [15].

Authors argue that this type of roadside installation might capture traffic parameters such as average speed, queue/congestion detection and journey times. However, it would be challenging to identify individual vehicles in specific lanes with roadside installation. They suggest routing cables perpendicular to the direction of travel to collect vehicle specific statistics.

2) Iron mine in Ma'anshan, China: Similar to [15], Liu et al. [1] also propose to use distributed acoustic sensing to monitor the vibrations from the vehicles. They carried out their field studies in the Ma'anshan iron mine in China. The fiber optic cables were installed on the shoulder of the road as shown in Fig. 24 covering $100 \mathrm{~m}$. Cement mortar was used to fix the optical fiber to the ground. The seismic signals induced by ore trucks and pickup trucks were recorded.

The space resolution was $1 \mathrm{~m}$, therefore there were 100 virtual sensors across the $100 \mathrm{~m}$ span. The scanning rate was $2500 \mathrm{~Hz}$. They collected data for the duration of two days using loaded trucks. The results were compared to an on-site video camera. Authors developed signal processing algorithms based on Wavelet theory to increase the signal-to-noise ratio (SNR) of the signals. The method achieves about $87 \%$ vehicle detection accuracy with a sample size of 38 . The vehicle count detection method becomes inaccurate when multiple vehicles are passing in series close to one another. Regarding

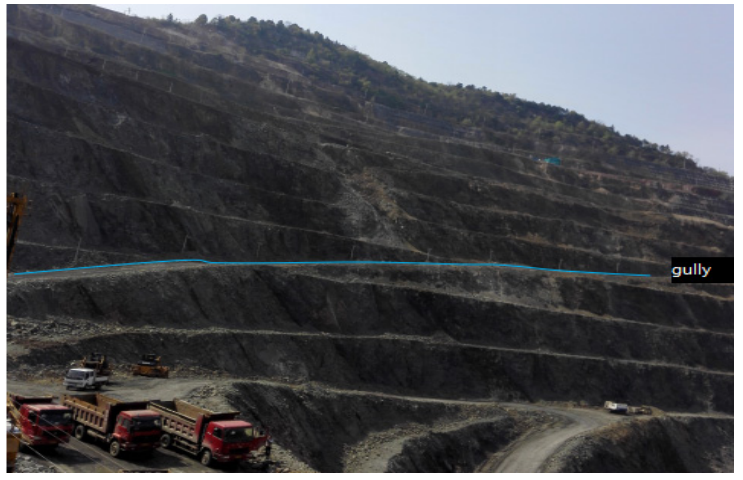

Fig. 24: Iron mine roadside cable installation in Ma'anshan, China [1].

the velocity calculation, their method stays within $6 \%$ error range.

\section{DISCUSSION}

\section{A. Sensitivity}

Perhaps the common challenge of the presented implementations was achieving a good coupling between the monitored environment and the sensor. For bridge instrumentation, the location (girder, soffit, support, abutment) and orientation of the sensors were key factors in producing a consistent detection method. Making those choices required extensive field trials and/or FEA since every bridge is structurally different. From an embedded in-pavement sensor installation perspective, the depth and orientation of the sensors played significant roles. For slabs of concrete, depths near the surface and near the bottom achieved the highest coupling. For asphalt roads, to the best of our knowledge, there has not been any FEA reported. Longitudinal sensors had the maximum sensitivity as opposed to vertical and transverse ones. Therefore, an array of longitudinal sensors can cover a given area with the least number of sensors making them a good practical choice in terms of cost. Regarding the distributed roadside installations, the highest coupling factor was reached when the cable was placed on the road shoulder.

\section{B. Resolution}

To detect individual axles, high spatial and temporal resolutions are needed. The size of the sensing elements should approximately not be larger than $50 \mathrm{~cm}$. This was one of the disadvantages of a long-gauge FBG design. Sampling frequency of the sensors should minimum be $500-1000$ Hz. Embedded and bridge implementations required sensor resolution of $1 \mu \epsilon$ to identify a typical passenger car. Systems operating at frequencies above a kilohertz with such resolution generally require expensive equipment. Advanced signal processing techniques can be developed as shown in [32] to improve sensor resolution values with relatively cheaper equipment. 


\section{Durability}

Fiber optic cables need appropriate packaging to survive harsh conditions. The packaging material needs extensive consideration as the selection can be a trade-off with the sensitivity of the sensor. Glass fiber reinforced polymer, polydimethylsiloxane, rubber, and plastic were among the chosen materials for robustness. In addition to sensor protection, the installation approach and the structure deterioration are important. A case of asphalt fiber installation based on microtrenching $(5-10 \mathrm{~cm}$ deep) was shown to crumble in about a year [33]. We need live real-world applications to gain insights about the durability of the overall system.

A roadway section is typically designed for 1 million equivalent single axle loads (ESALs) per year. Therefore any permanent roadway sensor installation should last the life of a typical roadway with a minimum of 15 million ESALs and a target of 50 million plus ESALs. The system should accommodate adjustments based on the design criteria of any section of the road it is incorporated into. For example, pavement depth and pavement construction materials should be modifiable depending on the expected ESALs to meet the durability requirement.

\section{Signal processing}

It was demonstrated by several of the studies that signal processing algorithms improved the accuracy. Adaptive filtering, Wavelet theory, and artificial neural networks were some of the tools utilized. Wavelet denoising was especially useful in denoising and capturing time-frequency response of the signals better than conventional methods.

\section{E. A unified solution}

Projects have demonstrated impressive proof of concepts with accuracy performances above $80 \%$ and in some cases above $90 \%$. However, we believe that solutions are local in space and in time. The experiments were only run for a few days on specific sites and only addressed a subset of the traffic parameters. There are some outstanding problems we would like to draw attention to. Typically, studies assume that every vehicle is made of two axles. If not they group the spikes (axles) in the signal if they are detected shortly after another. This inherently assumes that the speed of the vehicle is consistent across the measurement area which does not match the readily observed practices of vehicles in a real world environment. What is more, traffic can come to a full stop and erroneous axles can be grouped together. To the best of our knowledge the axle grouping issue has not been addressed yet. This problem can be attempted to solve by having a very dense sensing platform and an intelligence that is capable of tracking all axles at all times. Neural network based intelligence is a good candidate for this purpose. We believe that such a solution would integrate well with the upcoming connected electric autonomous vehicles providing a fully sensitive network of roads. To accomplish this we need a scalable solution.

Bridge sensing systems are tough to scale as every site would require a detailed FEA and extensive calibration.
Portable solutions might be a good option for certain private avenues but are probably not a viable one for public road adoption as they cause elevation in the road pavement surface by a few centimeters. Durability would be a colossal issue as it would directly be impacted by passing vehicles. It might also cause problems for snow plow trucks and vice versa. Surface installations are expensive to realize and maintain.

We need a global, user-friendly, consistent, durable, environment-friendly solution with high system availability that would work on bridges, in urban areas and in tunnels. Precast slabs of road pavement embedded with sensors that are manufactured at a facility would help with consistency and precision. This would prevent the potential inexact on-site installations, especially when workers are working in harsh conditions and attempting to complete the work under time constraints. Thinking about the self-driving vehicles of the future, sub-centimeter accuracy levels will need to be reached. Manufacturing precise and identical slabs would avoid the need for conventional fine tuning of the system parameters where strain values are correlated to weights and wheel positions. The networks of slabs would adopt the Plug and Play nature of the Internet. In other words, we need a general solution that is universally or semi-universally applicable, rather than point solutions that have to be independently redeveloped or calibrated for every location.

The use of a standardized precast pavement slab with embedded fiber optic sensors would reduce installation times, standardize delivery of fiber optic sensing into a near-universal design, and enable the simple replacement of any damaged or deteriorated sensor-embedded pavement sections with new, identical sections. Since the sensors would be embedded in the precast slab, durability would not be as much of an issue compared to surface sensors. Assuming that the sensor was housed in a rugged environment and had a longer life than the road, the bottleneck of the system would be the road and not the sensor. This type of design can truly be a global solution by having a single recipe and by being produced at a facility where large masses of it could be produced. To make the adoption easier, the number of embedded sensors per slab can gradually be increased, reducing the cost of the earlier installations.

\section{CONCLUSION}

It is evident that fiber optic sensors are well suited for traffic monitoring by capturing structural strain response. The existing implementations demonstrated great potential in counting vehicles, and estimating their speeds and weights with notable accuracy levels. One outstanding problem yet to be addressed is the axle grouping problem. Axle grouping problem arises from not knowing the speeds and number of axles a priori. We believe that the existing solutions are local in space and in time. The experiments were only run for a few days on specific sites and only addressed a subset of the traffic parameters.

We need a global, user-friendly, consistent, durable, environment-friendly solution with high system availability that would work on bridges, in urban areas and in tunnels. Precast slabs of road pavement embedded with sensors that are manufactured at a facility would help meet those demands. 


\section{ACKNOWLEDGMENT}

The authors would like to thank Integrated Roadways LLC and its partners for their support and insights.

\section{REFERENCES}

[1] H. Liu, J. Ma, W. Yan, W. Liu, X. Zhang, and C. Li, "Traffic flow detection using distributed fiber optic acoustic sensing," IEEE Access, vol. 6, pp. $68968-68980,2018$.

[2] Y. Bao, M. S. Hoehler, C. M. Smith, M. Bundy, and G. Chen, "Temperature measurement and damage detection in concrete beams exposed to fire using PPP-BOTDA based fiber optic sensors," Smart materials and structures, vol. 26, no. 10, p. 105034, 2017.

[3] S. Alamandala, K. Putha, R. S. Prasad, and P. R. Kumar, "FBG sensing system to study the bridge weigh-in-motion for measuring the vehicle parameters," in Proceedings of the Third International Conference on Microwave and Photonics (ICMAP). IEEE, 2018, pp. 1-2.

[4] D. Inaudi and B. Glisic, "Distributed fiber optic strain and temperature sensing for structural health monitoring," in Proceedings of the Third International Conference on Bridge Maintenance, Safety and Management, Porto, Portugal, 2006, pp. 16-19.

[5] C. E. Campanella, A. Cuccovillo, C. Campanella, A. Yurt, and V. Passaro, "Fibre Bragg grating based strain sensors: review of technology and applications," Sensors, vol. 18, no. 9, p. 3115, 2018.

[6] D. L. Presti, C. Massaroni, C. S. J. Leitão, M. D. F. Domingues, M. Sypabekova, D. Barrera, I. Floris, L. Massari, C. M. Oddo, S. Sales et al., "Fiber bragg gratings for medical applications and future challenges: A review," IEEE Access, vol. 8, pp. 156863-156888, 2020.

[7] B. Mollahasani Madjdabadi, "Experimental evaluation of a distributed fiber optic sensor for mining application," Ph.D. dissertation, University of Waterloo, 2016.

[8] K. Grattan and T. Sun, "Fiber optic sensor technology: an overview," Sensors and Actuators A: Physical, vol. 82, no. 1-3, pp. 40-61, 2000.

[9] A. Miliou, "In-fiber interferometric-based sensors: Overview and recent advances," in Photonics, vol. 8, no. 7. Multidisciplinary Digital Publishing Institute, 2021, p. 265.

[10] S. W. James and R. P. Tatam, "Optical fibre long-period grating sensors: characteristics and application," Measurement science and technology, vol. 14, no. 5, p. R49, 2003.

[11] V. Bhatia, "Applications of long-period gratings to single and multi-parameter sensing," Opt. Express, vol. 4, no. 11, pp. 457-466, May 1999. [Online]. Available: http://www.osapublishing.org/oe/abstract.cfm?URI=oe-4-11-457

[12] "Optical sensing instrumentation and software," Luna Innovations (Micron Optics, Inc.), User guide, 2017.

[13] X. Bao and L. Chen, "Recent progress in distributed fiber optic sensors," sensors, vol. 12, no. 7, pp. 8601-8639, 2012.

[14] A. H. Hartog, M. Belal, and M. A. Clare, "Advances in distributed fiberoptic sensing for monitoring marine infrastructure, measuring the deep ocean, and quantifying the risks posed by seafloor hazards," Marine Technology Society Journal, vol. 52, no. 5, pp. 58-73, 2018.

[15] A. Hall and C. Minto, "Using fibre optic cables to deliver intelligent traffic management in smart cities," in International Conference on Smart Infrastructure and Construction 2019 (ICSIC) Driving datainformed decision-making. ICE Publishing, 2019, pp. 125-131.

[16] L. Y. Mimbela, J. Pate, S. Copeland, P. M. Kent, J. Hamrick et al., "Applications of fiber optics sensors in weigh-in-motion (wim) systems for monitoring truck weights on pavements and structures." New Mexico. Dept. of Transportation, Tech. Rep., 2003.

[17] M. Lydon, S. E. Taylor, D. Robinson, P. Callender, C. Doherty, S. K. Grattan, and E. J. OBrien, "Development of a bridge weigh-in-motion sensor: performance comparison using fiber optic and electric resistance strain sensor systems," IEEE Sensors Journal, vol. 14, no. 12, pp. 4284 4296, 2014.

[18] M. Lydon, S. E. Taylor, C. Doherty, D. Robinson, E. J. O’Brien, and A. Žnidarič, "Bridge weigh-in-motion using fibre optic sensors," in Proceedings of the Institution of Civil Engineers-Bridge Engineering, vol. 170, no. 3. Thomas Telford Ltd, 2017, pp. 219-231.

[19] M. Lydon, S. E. Taylor, D. Robinson, A. Mufti, and E. Brien, "Recent developments in bridge weigh in motion (B-WIM)," Journal of Civil Structural Health Monitoring, vol. 6, no. 1, pp. 69-81, 2016.

[20] M. Lydon, D. Robinson, S. Taylor, G. Amato, E. Brien, and N. Uddin, "Improved axle detection for bridge weigh-in-motion systems using fiber optic sensors," Journal of Civil Structural Health Monitoring, vol. 7, no. 3, pp. 325-332, 2017.
[21] E. A. Oskoui, "Methods and applications of structural health monitoring on bridges," Ph.D. dissertation, University of Illinois at Chicago, 2019.

[22] S.-Z. Chen, G. Wu, D.-C. Feng, and L. Zhang, "Development of a bridge weigh-in-motion system based on long-gauge fiber bragg grating sensors," Journal of Bridge Engineering, vol. 23, no. 9, p. 04018063, 2018.

[23] T. Bao, S. K. Babanajad, T. Taylor, and F. Ansari, "Generalized method and monitoring technique for shear-strain-based bridge weighin-motion," Journal of Bridge Engineering, vol. 21, no. 1, p. 04015029 , 2016.

[24] G. Nosenzo, B. Whelan, M. Brunton, D. Kay, and H. Buys, "Continuous monitoring of mining induced strain in a road pavement using fiber bragg grating sensors," Photonic Sensors, vol. 3, no. 2, pp. 144-158, 2013.

[25] A. Batenko, A. Grakovski, I. Kabashkin, E. Petersons, and Y. Sikerzhicki, "Weight-in-motion (wim) measurements by fiber optic sensor: problems and solutions," Transport and Telecommunication, vol. 12, no. 4, pp. 27-33, 2011.

[26] A. Grakovski, A. Pilipovec, I. Kabashkin, and E. Petersons, "Weight-inmotion estimation based on reconstruction of tyre footprint's geometry by group of fibre optic sensors," Transport and Telecommunication, vol. 15 , no. 2, p. 97, 2014.

[27] M. A. Al-Tarawneh, "In-pavement fiber bragg grating sensors for weight in-motion measurements," Ph.D. dissertation, North Dakota State University, 2016.

[28] D. M. Karabacak, J. A. O’Dowd, L. J. Hopman, and J. M. Singer, "Asphalt embedded fibre optic weigh-in-motion technology," ICWIM8, p. $185,2019$.

[29] M. Fajkus, M. Fridrich, J. Nedoma, R. Kahankova, R. Martinek, E. Bednar, and J. Kolarik, "Pdms-fbg-based fiber optic system for traffic monitoring in urban areas," IEEE Access, vol. 8, pp. 127 648-127 658, 2020.

[30] K. Yuksel, D. Kinet, K. Chah, and C. Caucheteur, "Implementation of a mobile platform based on fiber bragg grating sensors for automotive traffic monitoring," Sensors, vol. 20, no. 6, p. 1567, 2020.

[31] G. Belitsky, V. Belitsky, and A. Liberson, "Mobile scales for traffic weighing based on optical fiber technology," ICWIM7, p. 26, 2016.

[32] D. Tosi, M. Olivero, A. Vallan, and G. Perrone, "Weigh-in-motion through fibre Bragg grating optical sensors," Electronics letters, vol. 46, no. 17 , pp. $1223-1225,2010$.

[33] J. Brodkin, "Google fiber's biggest failure: Isp will turn service off in Louisville," August 2019, [Online; posted 8-February-2019]. [Online]. Available: https://arstechnica.com/information-technology/2019/02/googlefiber-exits-louisville-after-shoddy-installs-left-exposed-wires-in-roads, 